\title{
ATRIAL ARRHYTHMIAS ABLATION IN A PATIENT POST MITRAL VALVE REPLACEMENT SURGERY AND COX-MAZE PROCEDURE
}

\author{
Hoang Trung Kien ${ }^{l}$, Vu Van Ba ${ }^{l}$, Le Tien Dung ${ }^{l}$, Nguyen Manh Hung ${ }^{l}$, \\ Do Duc Thinh ${ }^{1}$, Ngo Thi Hai Linh ${ }^{1,2,3}$, Tran Thuy Nguyen ${ }^{1,4^{*}}$
}

\section{ABSTRACT}

Objective: Cox Maze procedure is an effective surgical treatment of atrial fibrillation and it is often performed concomitantly with mitral valve surgery. However, some patients still experience atrial tachyarrhythmia post-surgery, which adversely affects heart function as well as their quality of life.

Method: We present the case of a 66-yearold female patient who underwent mitral valve replacement with a bio-prosthesis and Cox-Maze procedure; she was later admitted to our center after 14 months due to pneumonia and atrial tachycardia. An electrophysiology study/ intervention was performed.

Result: We successfully determined the mechanism and ablated the atrial tachyarrhythmia. The sinus rhythm was restored and was maintained 3 months post-procedure, with amelioration of the left ventricular function.

Conclusion: Electrophysiology study with electro-anatomy mapping can help to determine the mechanism of arrhythmia to guide successful ablation of post-operative arrhythmia.

Keywords: Cox Maze procedure, atrial fibrillation, Mitral valve surgery, Case report.

\section{INTRODUCTION}

Cox Maze procedure is an effective surgical treatment of atrial fibrillation and it is often performed concomitantly with mitral valve surgery. The combination of mitral valve replacement with Cox-maze procedure has been shown to be more effective than isolated valve surgery in maintaining sinus rhythm [1]. However, some patients still experience atrial tachyarrhythmia post-surgery, which adversely affects heart function as well as their quality of life. In this situation, radiofrequency ablation is one therapeutic option to completely eliminate the arrhythmia. This case report highlighted a patient who developed atrial arrhythmias after mitral valve replacement plus Cox-maze surgery and underwent successful radiofrequency ablation.

\section{CASE REPORT}

\section{Clinical presentation}

A 66 year - old female presented to our centre with a history hypertension, type II diabetes, and rheumatic mitral stenosis complicated with tricuspid regurgitation and atrial fibrillation. She underwent a cardiac surgery in September 2019 to replace the mitral valve with a bio-prosthesis Hancook $\mathrm{N}^{\circ} 27$ valve, combined with the insertion of a size 29 Medtronic annuloplasty ring, left atrial appendix closure, and bi-atrial Cox-maze IV. Post-operative course was uneventful.

1 Cardiovascular Center - E Hospital, Hanoi, Vietnam.

2 School of Clinical Medicine, Faculty of Medicine, The University of Queensland, QLD, Australia.

3 Department of Cardiology, The Prince Charles Hospital, QLD, Australia.

4 University of Medicine and Pharmacy - Vietnam National University, Hanoi, Vietnam

*Corresponding author: Nguyen Tran Thuy

Email: drtranthuyvd@gmail.com

Address: E Hospital, 87-89 Tran Cung, Hanoi, Vietnam, 10000

Vietnam National University, 114 Xuan Thuy, Hanoi, Vietnam, 10000

Received: 07 June $2021 \quad$ Accepted: 13 July 2021 
After the surgery, the patient still suffered from palpitation and dyspnoea. She was hospitalized multiple times due to decompensated heart failure. She was treated with a beta blocker (metoprolol 25mg) with no improvement in symptoms; she experienced complete heart block and atrial fibrillation when she was on digoxin $0.125 \mathrm{mg}$ daily.

In this current admission fourteen months after the operation, she was diagnosed with pneumonia. Her ECG showed a regular tachycardia thought to be atrial tachycardia (AT) with 2:1 ventricular response. Transthoracic echocardiography showed mildly dilated left ventricle (diastolic diameter of $55 \mathrm{~mm})$ and a reduced left ventricular ejection fraction (LVEF) of $36 \%$. We decided to first treat the pneumonia and electrophysiology study/intervention was performed once the infection settled.

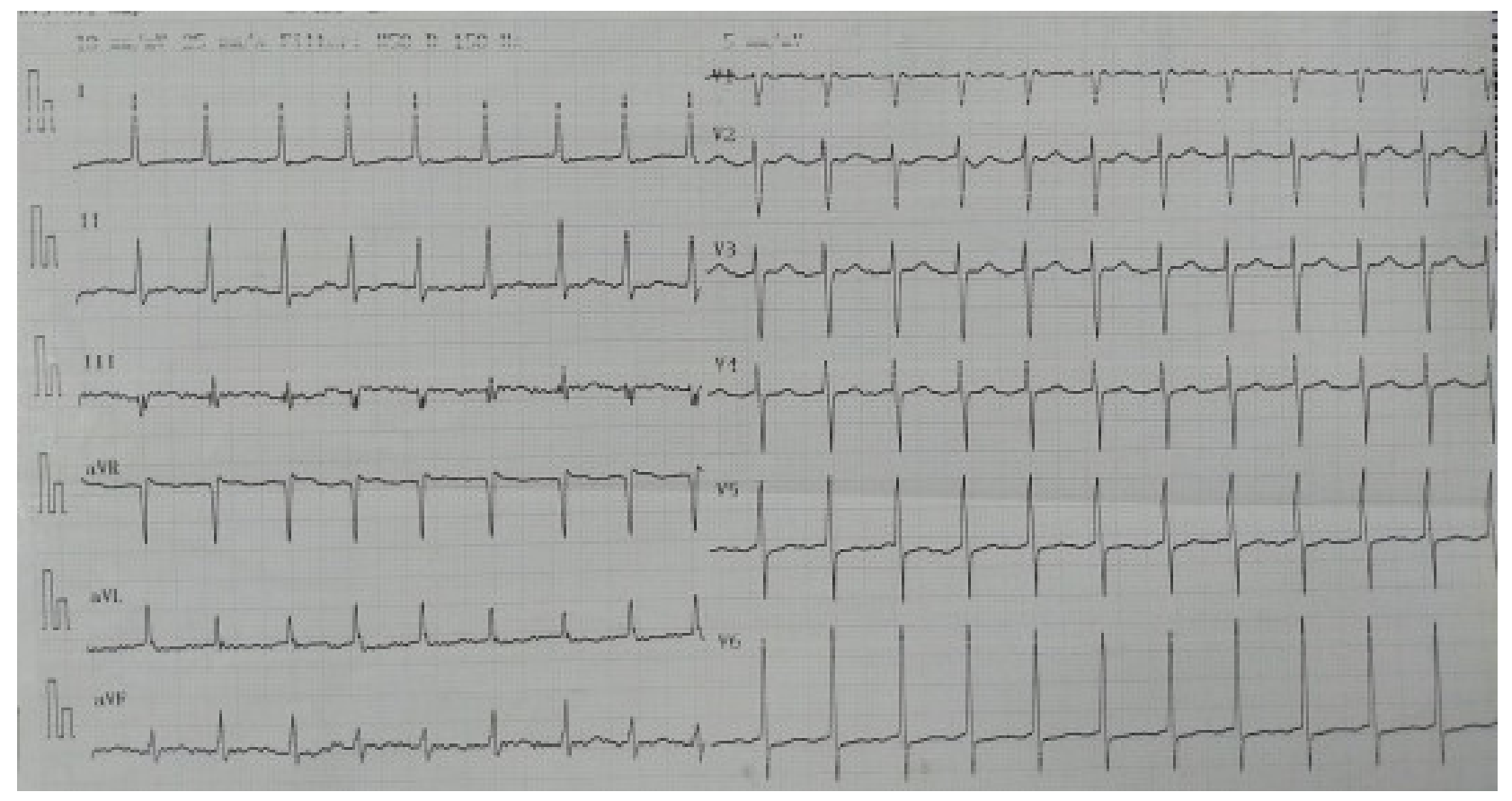

Figure 1. 12-lead ECG showed a narrow QRS tachycardia, with regular atrial waves and 2:1 ventricular response.

\section{Electrophysiology study (EPS) and RF ablation}

EPS and RF ablation were performed using the St. Jude EP Workmate System and St. Jude Ensite electro-anatomy mapping system. First, two 4-electrode catheters were placed in high right atrium and right ventricular apex respectively and a deca-polar electrode catheter was placed in the coronary sinus (CS). The electrograms showed the first atrial tachycardia with an atrial tachycardia cycle length (CL) of 230ms, 2:1 ventricular response, and concentric atrial activation sequence (figure 2A) which spontaneously changed to the second atrial tachycardia with atrial CL of $220 \mathrm{~ms}$ and eccentric atrial activation sequence (earliest at distal CS - figure 2B). 


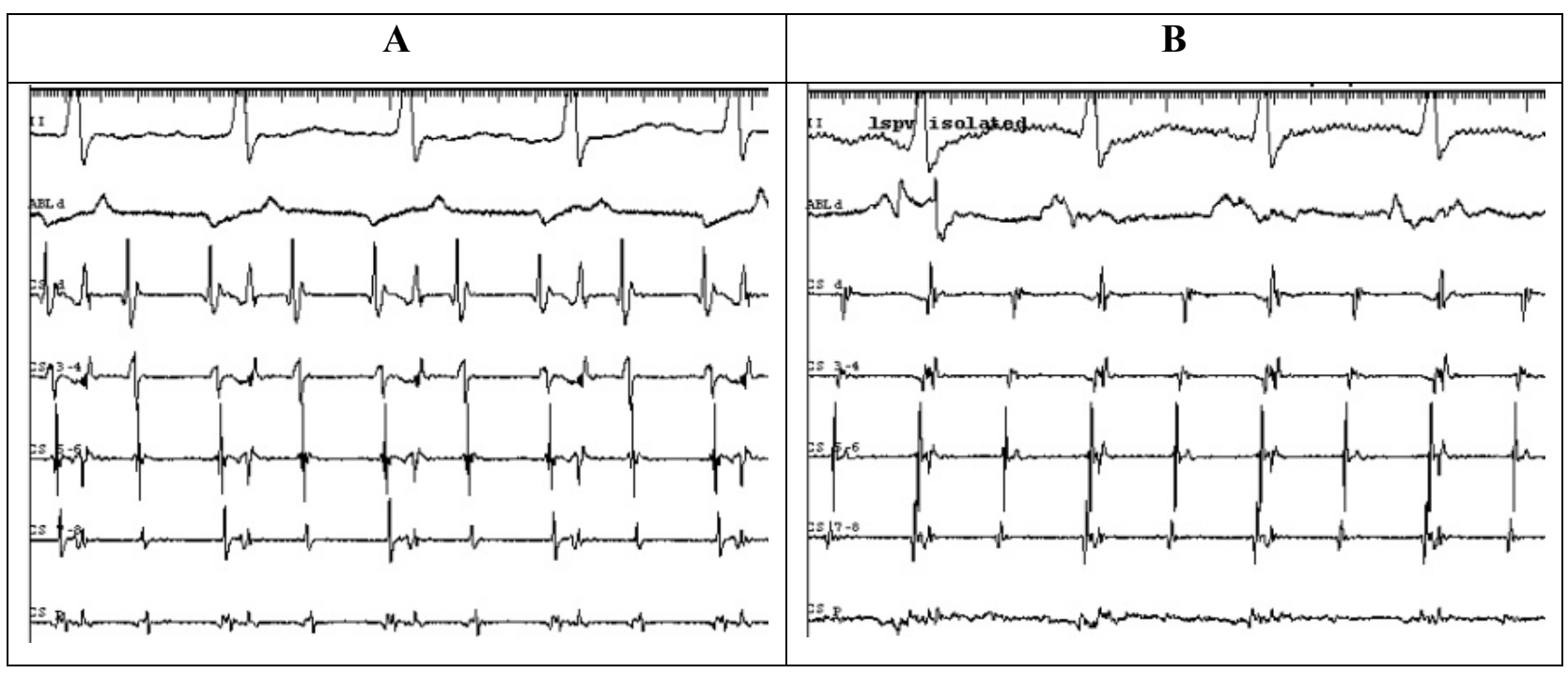

Figure 2. The two forms of atrial tachycardia.

We decided to perform an atrial trans-septal puncture to approach the left atrium. With activation mapping, we identified that the focal AT originated from the anterior part of left inferior pulmonary vein, near the mitral line; the right pulmonary veins and left atrial posterior wall were isolated after the Coxmaze procedure; the left pulmonary veins were re-connected (figure 3). After successfully ablated this focal AT (white dots in figure 3), the atrial activation sequence returned to what was seen during the first atrial tachycardia. We then successfully re-isolated the left pulmonary veins (yellow dots in figure 3 ).

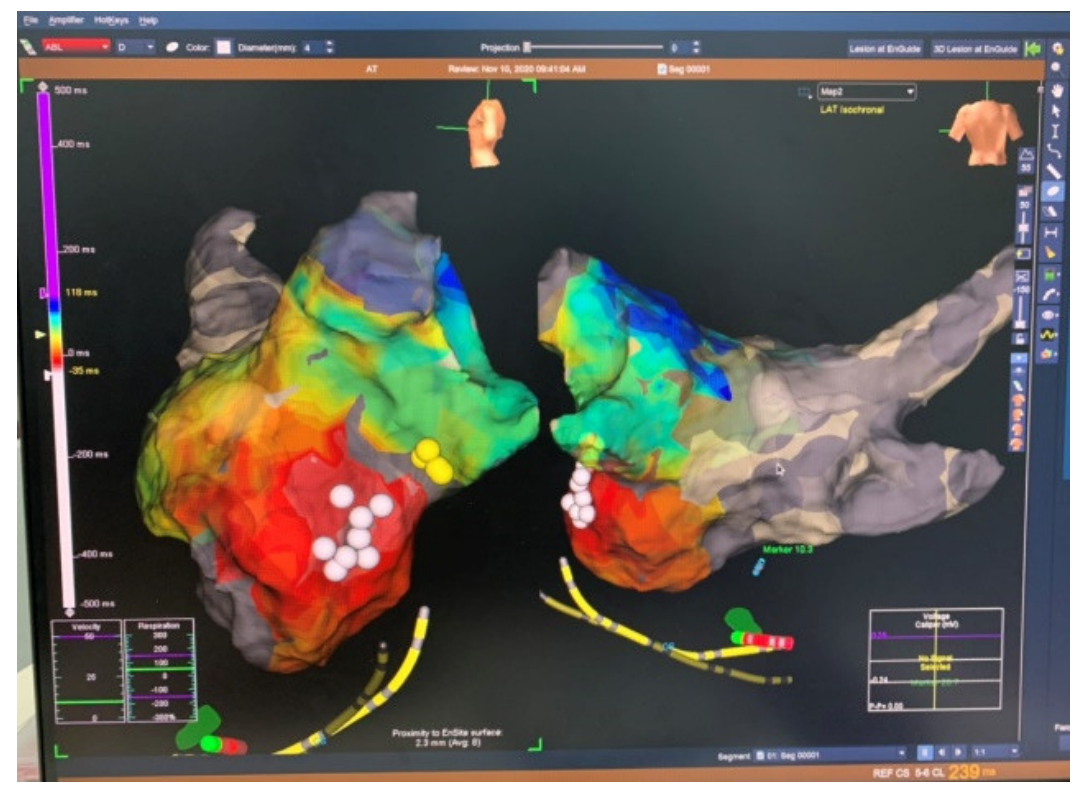

Figure 3. 3-dimensional activation mapping of the left atrium during the second tachycardia. We found that both the right pulmonary veins and the posterior wall of left atrium were completely isolated (grey region). The white dots were target area for the ablation of the second atrial tachycardia ablation, the yellow dots were target area for left pulmonary veins isolation. 
We then examined the first atrial tachycardia and with propagation mapping, the tachycardia was diagnosed as a typical cavotricuspid isthmus-dependent atrial flutter (figure 4A). Ablation of the CTI terminated the flutter and transient junctional rhythm was present, followed by sinus rhythm in the end of the procedure (figure 4B).

A

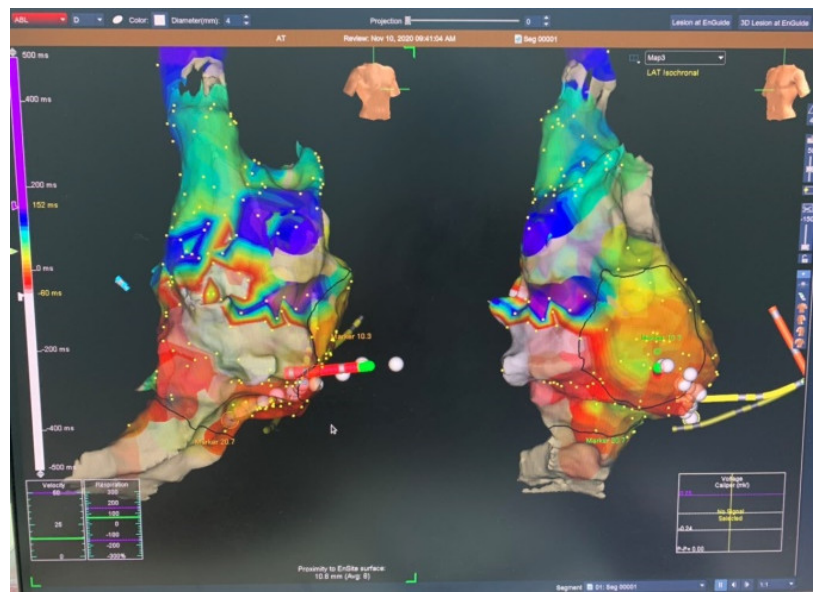

B

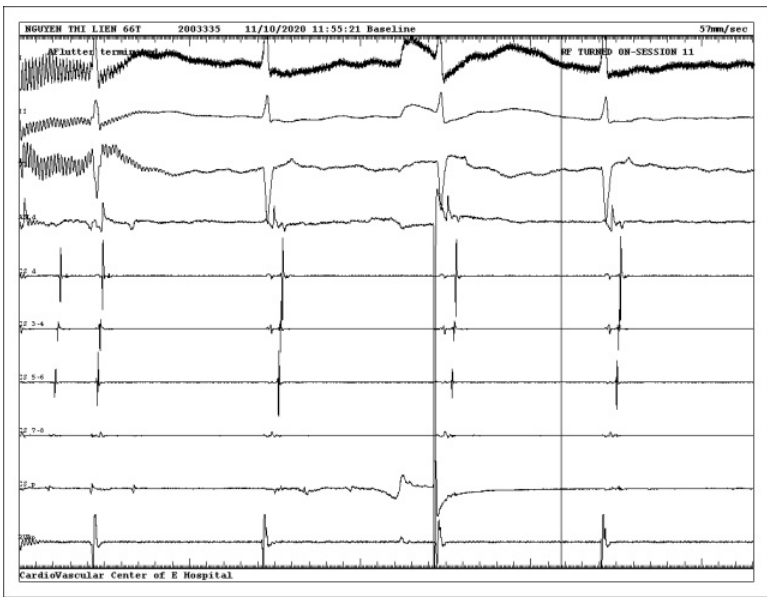

Figure 4. Activation mapping of the first atrial tachycardia showed typical atrial flutter. The flutter was terminated when ablating the CTI, with the presence of junctional rhythm.

Patient's symptoms were improved after the procedure. We continued metoprolol (at the time of procedure, amiodarone was not available in our country). ECG at discharge showed the sinus rhythm, with slightly prolonged QT interval, probably due to quinolone (figure 5). After 3 months, the patient remained in sinus rhythm, with the amelioration of left ventricular function.

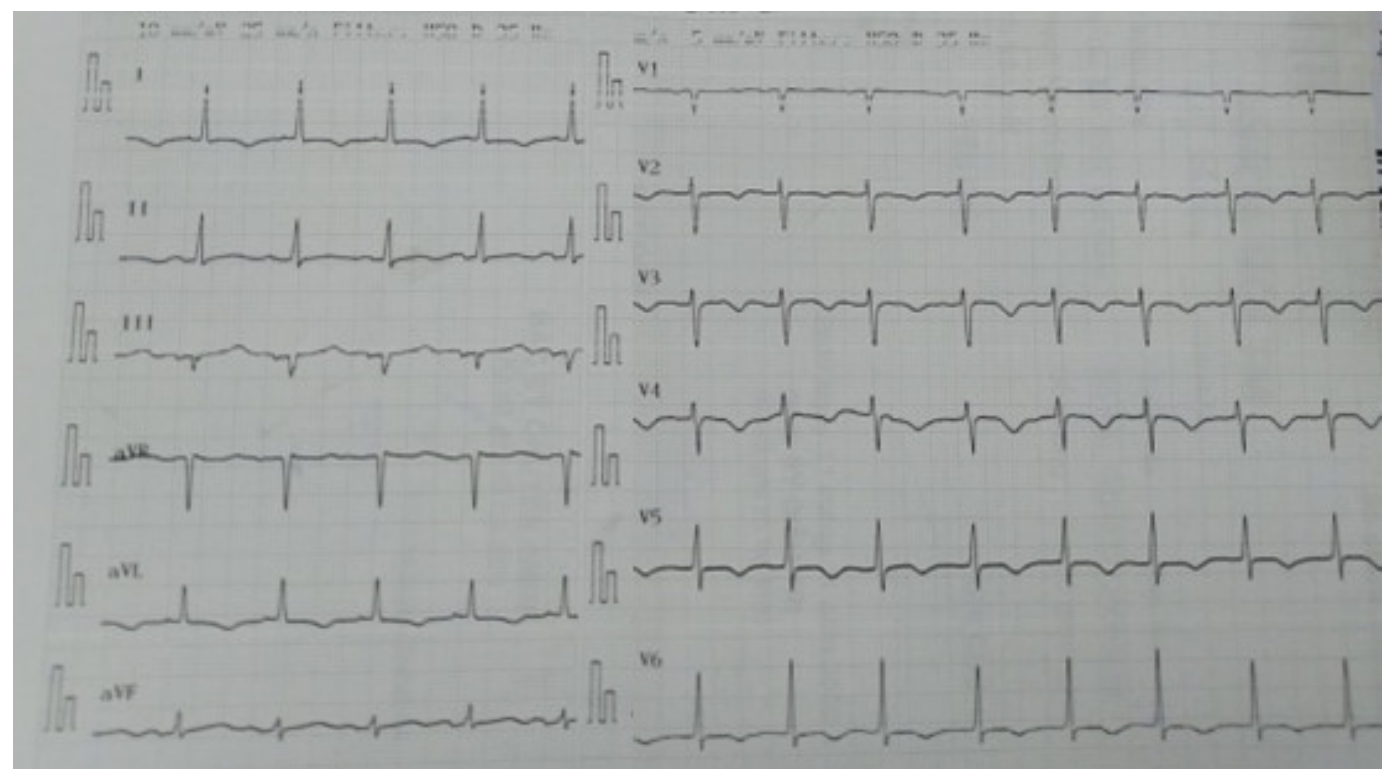

Figure 5. ECG at discharge. 


\section{DISCUSSION}

Cox-maze procedure is effective for the management of atrial fibrillation, with normal sinus rhythm restored in approximately 91-93\% of patients at 1 year, a rate that fell to $71-81 \%$ at 5 years[2-4]. Main risk factors for atrial fibrillation recurrence included time to onset of atrial fibrillation, atrial size on echocardiography, f-wave amplitude on ECG and average heart rate in Holter ECG [2,5,6]. The study by Kajiyama et al. on 11 patients undergoing the electrophysiology study for atrial arrhythmias after Cox-maze procedure showed that the mechanism of arrhythmias is mainly local injury as well as re-connection in the area around the mitral annulus [7]. With the support of the electro-anatomy mapping system, we can directly check if the pulmonary veins have really been isolated. Moreover, the results of EPS can be reported to cardiac surgeons, which may help to improve the successful rate of future Cox-maze procedures [7]. Regarding the safety of ablation, there is always a risk of damage to the prosthetic valve during left atrial catheterization. Nevertheless, ablation is still a safe procedure if it is performed carefully in experienced centres [8,9].

In Vietnam, ablation of atrial arrhythmias in patients with prosthetic valves has not been done frequently. However, in the future, with many more patients with mitral valve disease undergoing cardiac surgery, ablation of post-operative atrial arrhythmias will provide an effective and safe therapeutic option that could help to relieve symptoms and improve long-term prognosis.

\section{CONCLUSION}

Cox-Maze procedure is an effective treatment for lone atrial fibrillation as well as for atrial fibrillation associated with valvular disease, but there is still a high incidence post-operative atrial arrhythmia. Electrophysiology study with electro-anatomy mapping can help to determine the mechanism of arrhythmia to guide successful ablation of arrhythmia.

Consent: The author/s confirm that written consent for submission and publication of this case report including image(s) and associated text has been obtained from the patient in line with COPE guidance.

\section{REFERENCES}

1. Jessurun ER, van Hemel NM, Defauw JJ, Brutel De La Rivière A, Stofmeel M a. M, Kelder JC, et al. A randomized study of combining maze surgery for atrial fibrillation with mitral valve surgery. J Cardiovasc Surg (Torino). 2003 Feb;44(1):9-18.

2. Chen M-C, Chang J-P, Chen Y-L. Surgical treatment of atrial fibrillation with concomitant mitral valve disease: an Asian review. Chang Gung Med J. 2008 Dec;31(6):538-45.

3. Bando K, Kobayashi J, Kosakai Y, Hirata M, Sasako Y, Nakatani S, et al. Impact of Cox maze procedure on outcome in patients with atrial fibrillation and mitral valve disease. J Thorac Cardiovasc Surg. 2002 Sep;124(3):575-83.

4. Damiano RJ, Schwartz FH, Bailey MS, Maniar HS, Munfakh NA, Moon MR, et al. The Cox maze IV procedure: Predictors of late recurrence. The Journal of Thoracic and Cardiovascular Surgery. 2011 Jan 1;141(1):113-21.

5. Kim KC, Cho KR, Kim Y-J, Sohn D-W, Kim K-B. Long-term results of the CoxMaze III procedure for persistent atrial 
fibrillation associated with rheumatic mitral valve disease: 10-year experience. Eur J Cardiothorac Surg. 2007 Feb;31(2):261-6.

6. Takagaki M, Yamaguchi $\mathrm{H}$, Ikeda $\mathrm{N}$, Yamakage H, Nakamura H, Kadowaki T, et al. Risk Factors for Atrial Fibrillation Recurrence After Cox Maze IV Performed Without Pre-exclusion. Ann Thorac Surg. 2020 Mar;109(3):771-9.

7. Kajiyama T, Kondo Y, Ueda M, Nakano M, Nakano M, Watanabe $M$, et al. Catheter ablation of atrial tachyarrhythmias after a Maze procedure: A single center experience. J Cardiol Cases. 2018 Dec 20;19(3):89-92.
8. Lang CC, Santinelli V, Augello G, Ferro A, Gugliotta F, Gulletta S, et al. Transcatheter radiofrequency ablation of atrial fibrillation in patients with mitral valve prostheses and enlarged atria: safety, feasibility, and efficacy. J Am Coll Cardiol. 2005 Mar 15;45(6):868-72.

9. Zipse MM, Nguyen DT. Ablation of Atrial Fibrillation in a Patient with a Mechanical Mitral Valve. Cardiac Electrophysiology Clinics. 2016 Mar 1;8(1):155-9. 